\title{
EXPERIMENTAL DESIGNS FOR SYSTEM ASSESSMENT AND IMPROVEMENT WHEN NOISE FACTORS ARE CORRELATED
}

\author{
Susan M. Sanche" \\ School of Business Administration \\ University of Missouri-St. Louis \\ 8001 Natural Bridge Road \\ St. Louis, Missouri 63121-4499, U.S.A.
}

\begin{abstract}
Taguchi's strategy for tolerance design provides insight into the transmission of variability from noise factors to the system response. Under assumptions of independent noise factors, simple orthogonal designs and analysis allow for straightforward assessment of system sensitivity and evaluation of alternative system configurations. However, in certain cases the assumptions of independent noise factors are invalid. How, then. should an experiment be designed to allow similar sensitivity analysis? We illustrate our results for a model of a simple circuit, where internal noise factors may be positively correlated due to environmental conditions.
\end{abstract}

\section{INTRODUCTION}

Response surface metamodels allow an analyst to examine many scenarios without rerunning the simulation, thus ways of efficiently developing response surface metamodels are of interest. Response surface metamodels can also be used in conjunction with Taguchi's robust design strategy to select the preferred system configuration, and to assess the system's performance and attribute the overall system variance to noise factors for a specific parameter configuration (Myers et al., 1992; Sanchez et al.. 1993) This also allows the analyst to examine several types of potential changes to the system to see how they will benefit the overall system performance. For an overview of the application of robust design to discrete event simulation, see the tutorial by Sanche\% (1994) in this volume. We begin by bricfly discussing the concepts pertinent to this paper.

Before rumning an experiment, the analyst specifies a performance measure which is of particular interest, such as the average time in system for a queueing network. Then, perhaps in conjunction with others familiar with the process, he/she identifies factors which are thought to affect this performance measure. The factors are classified as parameters (or decision factors) if they are controllable in the real world setting, and noise factors if they are uncontrollable or controllable only at great expense. Noise factors can either be internal (based on endogenous characteristics of the system) or external (resulting from exogenous effects). For example, if you are modeling a manufacturing system, then decision factors such as the numbers and types of machines, shop floor layout, batch size, and material handling system might represent decision factors; machine breakdowns and processing times are internal noise factors, and customer demand patterns or supplier lead times are cxternal noise factors.

In the parameter design (or robust design) stage. one conducts a designed experiment to gather information about the expected system performance (across the noise space) and then evaluates performance by using a loss function, which takes into account both the mean and the variability of the performance measure. A way of accomplishing this is to build response surface models of both the performance mean and (log of) variability, and combine the results assuming a quadratic loss function. The ideal system configuration is one which results in a mean performance equal to the target, $\tau$, and a performance variability of zero. In practice, the ideal may not be attainable. The loss function serves to trade off average deviation from the target with consistency of the output, and the 'best' system is often not that associated with the best mean performance.

The final stage in Taguchi's framework is called tolcrance design (Taguchi 1986, 1987; see also D'Errico and Zaino, 1988.) In this stage, the analyst evaluates the overall system performance, attributes variation in the response to variation in the noise factors. and determines whether or not upgrades or downgrades in the consistency of component parts or other sources of noise are worthwhile. Typically only main effects are 
considered, and the noise factors are assumed to be mutually independent. This assumption is met (by design) during the experiment, but it may be that some of these factors are correlated in the real world sctting under nonexperimental (e.g., normal operating) conditions. If so, this raises several questions: (1) What affect does correlation have on the tolerance analysis results? (2) How can the regression models devcloped under an independence assumption be used to evaluation the system when noise factors are correlated? (3) Are there better alternatives for the experimental design if correlation is known to exist a priori? We address the first two questions in Section 2 , and the last question in Section 3.

\section{USE OF METAMODELS WHEN SOME NOISE FACTORS ARE CORRELATED}

Let $Y$ denote the performance measure of interest, $\left\{W_{1}, \ldots, W_{k}\right\}$ denote the noise factors controlled during the course of the experiment, and let $\mu_{i}$ and $\sigma_{i}^{2}$ denote the mean and variance of $W_{i}(i=1, \ldots, k)$. Then, assuming that the noise factor ranges are small enough that a linear model is appropriate, we have

$$
Y=\beta_{0}+\beta_{1} W_{1}+\beta_{2} W_{2}+\ldots+\beta_{k} W_{k} .
$$

A orthogonal experimental design (often a factorial or fractional factorial) is used for collecting data regarding the process, and a linear metamodel is fit:

$$
\hat{Y}=\hat{\beta}_{0}+\hat{\beta}_{1} W_{1}+\hat{\beta}_{2} W_{2}+\ldots+\hat{\beta}_{k} W_{k}
$$

Assuming that the $W_{i}$ are independent and normally distributed, then the metamodel of equation (2) can be used to determine the overall system mean and variance by treating the cstimated coefficients as constants:

$$
\begin{aligned}
\mu_{Y} & \approx \hat{\beta}_{0}+\hat{\beta}_{1} \mu_{1}+\hat{\beta}_{2} \mu_{2}+\ldots+\hat{\beta}_{k} \mu_{k} \\
\sigma_{Y}^{2} & \approx \hat{\beta}_{1}^{2} \sigma_{1}^{2}+\hat{\beta}_{2}^{2} \sigma_{2}^{2}+\ldots+\hat{\beta}_{k}^{2} \sigma_{k}^{2}
\end{aligned}
$$

The term $\sigma_{t ; i}^{2} \equiv \hat{\beta}_{i}^{2} \sigma_{i}^{2}$ is called the transmitted variance of noise factor $i$. The ratio $\sigma_{t ; i}^{2} / \sigma_{Y}^{2}$ is the proportion of the variability in the response that is attributable to $W_{i}$. Potential changes to the noise factors (which affect the variance alone, not the mean) can then be evaluated by considering the reduction on the overall performance variability $\sigma_{Y}^{2}$ and the corresponding reduction in cost.

Now suppose that two of the $k$ noise factors $\left(W_{1}\right.$ and $W_{2}$ ) are not independent in the real world, but have a correlation of $\rho$. (All other pairs of variables are independent.) In this case, the value of
$\mu_{Y}$ remains unchanged from that computed in equation (3). However, the overall variance is no longer correct. The true value is

$$
\begin{aligned}
\sigma_{Y}^{2} \approx & \hat{\beta}_{1}^{2} \sigma_{1}^{2}+\hat{\beta}_{2}^{2} \sigma_{2}^{2}+2 \rho \hat{\beta}_{1} \hat{\beta}_{2} \sigma_{1} \sigma_{2} \\
& +\hat{\beta}_{3} \sigma_{3}^{2}+\ldots+\hat{\beta}_{k}^{2} \sigma_{k}^{2}
\end{aligned}
$$

which is greater than the value in equation (4) if $\rho>0$ and less than this value if $\rho<0$. This means that the overall estimate of system variability and the transmitted variance proportions are different than those calculated assuming independence.

If the $W_{i}$ are truly independent, the transmitted variances completely separate the effects for the various noise factors. This does not occur when $W_{1}$ and $W_{2}$ are correlated, although orthogonal experimental designs might be desirable because of the increased precision of the estimated coefficients. However, the results are no longer separable in the real world. Rather than computing separate transmitted variances for both factors, we use a joint transmitted variance:

$$
\sigma_{t ; 1,2}^{2}=\hat{\beta}_{1}^{2} \sigma_{1}^{2}+\hat{\beta}_{2}^{2} \sigma_{2}^{2}+2 \rho \hat{\beta}_{1} \hat{\beta}_{2} \sigma_{1} \sigma_{2}
$$

Potential changes to the system components can then be assessed by determining the resulting changes in the joint transmitted variance and the overall system variance.

Table 1: Component Distribution Models

\begin{tabular}{c|r|r|r} 
Component & Mean & Std. Dev. & $\begin{array}{r}\text { Percent } \\
\text { of Mean }\end{array}$ \\
\hline L & 0.004 & 0.0008 & $20 \%$ \\
R & 10 & 1.0 & $10 \%$ \\
V & 100 & 5.0 & $5 \%$ \\
f & 50 & 5.0 & $10 \%$ \\
\hline
\end{tabular}

We now illustrate the effects of incorrectly assuming independence for a circuit example discussed in Ramberg et al. (1991). Consider an electrical circuit with a performance measure of current $I$ (in amps) and a target $\tau=10$ amps. The analytical expression is:

$$
I=\frac{V}{\sqrt{R^{2}+(2 \pi f L)^{2}}}
$$

where $V$ is the voltage (in volts), $R$ is the resistince (in ohms), $f$ is the frequency (in Hert $\%$ ). and $L$ is the inductance (in Hemries). The variation in $L$ and $R$. from their nominal means, as well as the values of $V$ and $f$ from the environments in which the circuit will opcrate, are all sources of noise. 
Although an analytical expression for the circuit performance is known, assessing its performance when the component characteristics are random variables is not mathematically tractable. One way of addressing the problem is Monte Carlo sinulation: an efficient alternative is the use of a designed experiment. Tables 1 and 2 (from Ramberg et al.. 1991) show the distribution models of the four noise factors, and the experimental design and results for a $2^{4-1}$ half-fraction experiment. The fitted metamodel for the current is provided in equation (8).

$$
\begin{aligned}
I \approx & 10.145-40.86 L-0.9853 R \\
& +0.1005 V-0.00329 f
\end{aligned}
$$

From this metamodel, the overall mean and variance are estimated to be 10.014 and 1.225 . respectively.

Table 2: Results of Designed Experiment

\begin{tabular}{c|crcc} 
Response & \multicolumn{4}{|c}{ Natural Levels } \\
I & L & R & \multicolumn{1}{c}{ V } & F \\
\hline 10.5026 & 0.0032 & 9 & 95 & 45 \\
10.3807 & 0.0048 & 9 & 95 & 55 \\
8.5931 & 0.0032 & 11 & 95 & 55 \\
8.5714 & 0.0048 & 11 & 95 & 45 \\
11.5796 & 0.0032 & 9 & 105 & 55 \\
11.5362 & 0.0048 & 9 & 105 & 45 \\
9.5133 & 0.0032 & 11 & 105 & 45 \\
9.4387 & 0.0048 & 11 & 105 & 55 \\
\hline
\end{tabular}

Now we compare the system variance estimates and noise factor assessments under various assumptions regarding the independence of the noise factors. Three situations are shown in Table 3: results computed under (1) independence, (2) when the correlation between $L$ and $R$ is .9 , and (3) when the correlation between $L$ and $R$ is -.9. Even though the inductor $L$ has a very small impact on overall system variability in the independent case, the overall result underestimates the true systcm variance by $5 \%$ if the true correlation is .9 , and overestimates the true system variance by $5 \%$ if the true correlation is -.9 .

The use of the metanodel information for systcm improvement analysis deserves a bit more discussion. Analysis for any changes regarding the variance of $W_{i}$ $(i \neq 1,2)$ proceeds just as before. The cost for changing $\sigma_{i}^{2}$ can be compared to the reduction in expected loss (or risk), where

$$
R \equiv E[\text { loss }]=c\left[\left(\mu_{Y}-\tau\right)^{2}+\sigma_{Y}^{2}\right]
$$

The reduction in risk one can achieve by altering $\sigma_{i}^{2}$
Table 3: Results for Independent and Correlated

\begin{tabular}{|c|c|c|c|}
\hline & \multicolumn{3}{|c|}{ Transmitted Variances ( $\%$ of MSE) } \\
\hline & $\rho=0$ & $\rho=.9$ & $\rho=-.9$ \\
\hline $\mathrm{L}$ & $.001(00.1)$ & & \\
\hline & & $1.030(80.3)$ & $0.914 \quad(78.3)$ \\
\hline $\mathrm{R}$ & $.971 \quad(79.3)$ & & \\
\hline$\overline{\mathrm{V}}$ & $.253 \quad(20.6)$ & $.253 \quad(19.7)$ & $.253 \quad(21.7)$ \\
\hline $\mathrm{f}$ & $.000 \quad(0.0)$ & $.000 \quad(0.0)$ & .000 \\
\hline tot & $1.225(100.0)$ & $1.283(100.0)$ & $1.167(100.0)$ \\
\hline
\end{tabular}
Components Using Metamodels From Orthogonal Designs

by a factor of $\gamma$ is then

$$
\Delta R=c\left[\sigma_{t, i}^{2}-\gamma^{2} \sigma_{t, i}^{2}\right]=c \sigma_{t, i}^{2}\left(1-\gamma^{2}\right)
$$

If $\Delta R$ is greater than the cost of improvement (i.e.. if $\gamma<1$ ), then the improvement is cost-effective. If $\gamma>1$, we are considering relaxing the standard for a particular component. This results in lower expected loss as long as $\Delta R$ is less than the savings in component cost.

For the correlated factors $W_{1}$ and $W_{2}$. what types of changes are possible? First, suppose that the variance in both these factors results from some common cause not explicitly included as a factor. For the circuit example, perhaps $V$ and $f$ are positively correlated because of some environmental factors. Attention to these factors might improve both $\sigma_{1}$ and $\sigma_{2}$ ly multiplying them by a factor of $\gamma<1$, but leave the correlation between $W_{1}$ and $W_{2}$ unchanged. Then

$$
\Delta R=c\left[\sigma_{t ; 1,2}^{2}-\gamma^{2} \sigma_{t ; 1,2}^{2}\right]=c \sigma_{t ; 1,2}^{2}\left(1-\gamma^{2}\right)
$$

and, as before, this is cost-effective as long as $\Delta R$ is greater than the cost of improvement.

Second, suppose that changes in the real world system leave the variances unaltered but change the correlation. This might occur if, for instance, component parts were initially purchased from the same vendor but are now purchased from two separate vendors. In gencral, suppose the correlation $\rho$ would change to a new value $\rho^{\prime}$. If the cost of such a switch is less than

$$
\Delta R=2 \hat{\beta}_{1} \hat{\beta}_{2} \sigma_{1} \sigma_{2}\left(\rho-\rho^{\prime}\right)
$$

then the switch is cost-effective.

The results in this section can be generalized in a straightforward manner to situations where more than two variables are correlated. The overall system evaluation remains easy to compute. However. as the 
amount of dependence in the noise factors increases (in terms of the correlation structure), the separable effects are fewer. This can severely limit the utility of the tolerance design analysis: one of the benefits cited for Taguchi's approach is its simplicity. Therefore, wc recommend analysis using correlation structures only if correlations are strongly apparent in the real world.

\section{ALTERNATIVE DESIGNS}

In some situations, the correlation in the real world may restrict the choicc of the design. The work described in this paper was motivated by a practical application: we wished to investigate the sensitivity of a forecasting model to various cnvironmental factors (Smith et al., 1994). A metamodel of home mortgage losses was developed for a major California financial institution, and a simulation option allowed the clients to examine alternative scenarios. The final model consisted of three subcomponents. First, a Markovian structure was fit to forecast the probability of transition betwecn five states on a yearly basis over the remaining lifetime of the portfolio (30 years). Three states were non-absorbing: a mortgage could be 'Current,' '30-89 days delinquent,' or ' $90+$ days delinquent.' The two absorbing states were 'Paid-in-Full' and 'Defaulted.' The second component further split the defaulted loans, into those resulting in a monetary loss and those for which sufficient equity was available to cover the mortgage balance (e.g., if the institution held the first mortgage of a home, and foreclosure occurred because the homeowner defaulted on a second mortgage.) Finally, for those loans resulting in a nonzero loss, a simple model predicted the percentage of the remaining balance lost. Simulation of the portfolio's performance allowed the client to specify changes in three envirommental components (interest rates, unemployment rates, and market appreciation rates) for years $1-5$ and $6+$ which varied by four different geographic locations.

In this situation, the metamodel was built from observational, rather than experimental, data. The noise factors in the observational data (interest rates, uncmployment rates, and market appreciation rates) were highly correlated. Although the metamodel could have becn analyzed by constructing a factorial design, we felt this was inappropriate because (a) certain combinations of the factors were not likely to occur, thus the purpose of sampling at levels such as $\mu \pm \sigma$ in order to get the same variability in the sampling distribution as in the underlying normal distribution was lost, and (b) the metamodel was not constructed using similar combinations. The results would be highly suspect for such dramatic extrapolattions.

For other systems, it might be that a factorial or fractional factorial design could not be conducted over the entire range of interest. For example, a queueing system might be unstable if all noise factors were held at their high levels. If this situation was unlikely to occur in practice because of correlation among the variables, then a sampling scheme which made use of the underlying dependence struc:ture would seem more appropriate.

Once again, for simplicity, we assume that the noise factors are normally distributed but only the first two $\left(W_{1}\right.$ and $\left.W_{2}\right)$ are correlated. We also assume, without loss of generality, that $\mu_{i}=0$ and $\sigma_{i}=1$ $(i=1, \ldots, k)$. (This is less restrictive than it sounds: it corresponds to standardizing all our noise factors by subtracting the mean and dividing by the standard deviation before conducting the experiment.) The joint distribution of $W_{1}$ and $W_{2}$ is then a bivariate normal. Letting $W_{1}$ be on the $x$-axis and $W_{2}$ on the $y$-axis, the contours of the bivariate distribution are elliptical, satisfying

$$
x^{2}-2 \rho x y+y^{2}=c^{2}
$$

as long as $|\rho|<1$ (Dudewicz and Mishra. 1987).

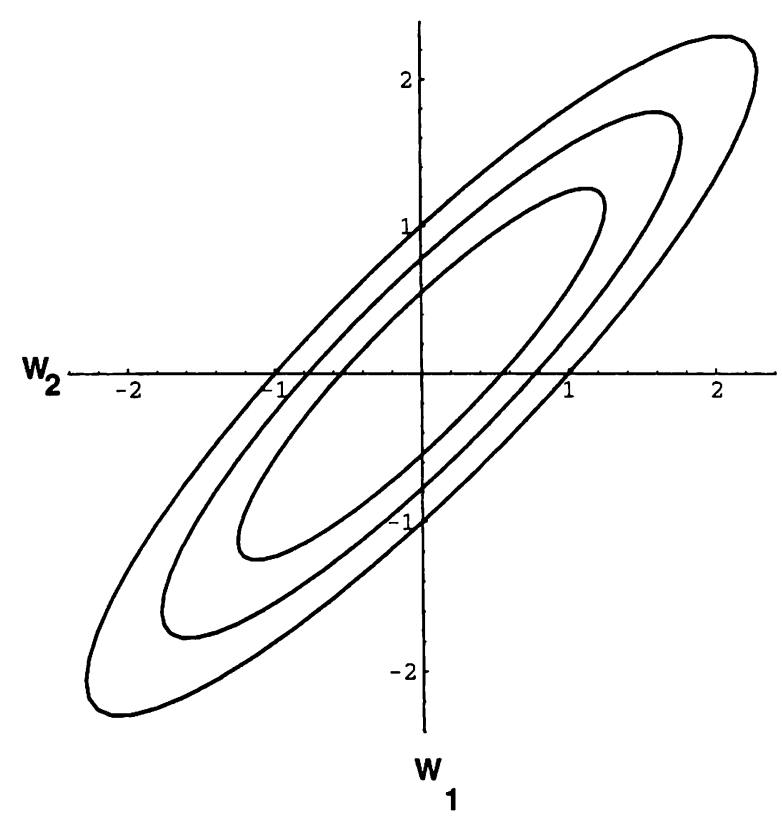

Figure 1: Density Contours for Bivariate Standard Normal with Correlation Coefficient $\rho=.9$

If $\rho=0$ then equation (9) reduces to a circle with radius $c$ centered about the origin. If $\rho \neq 0$ then the major axis of the ellipse falls on the line $y=x$ and the minor axis is along the line $y=-1 / x$. as 
shown in Figure 1. If we ran our experiment as if $W_{1}$ and $W_{2}$ were independent, then a 2-level factorial would results in sampling at the 4 points $( \pm 1, \pm 1)$. These correspond to sampling each of the two factors at $\mu \pm \sigma$, so that the discrete sampling distribution for $W_{1}$ and $W_{2}$ have the same mean and variance as the marginal distributions. An alternative, which looks much more reasonable in the prescnce of correlation, is to sample at points on one of the elliptical contours.

From equation (9) and basic geometry (Beyer. 1987) onc can compute coordinates of the major and minor axial points for given $c$. These are given in equations (10) and (11) for $\rho>0$, again for the case when standardized units are used for both noise factor designs. (If $\rho<0$ then equation (10) gives the minor axial points and equation (11) gives the major axial points.)

Major Axial Points

$$
\pm\left(\frac{c}{\sqrt{2(1-\rho)}}, \frac{c}{\sqrt{2(1-\rho)}}\right)
$$

Minor Axial Points

$$
\pm\left(\frac{-c}{\sqrt{2(1+\rho)}}, \frac{c}{\sqrt{2(1+\rho)}}\right)
$$

If we sample at the four axial points, then the variance of the sampling distribution for either $W_{1}$ or $W_{2}$ will be

$$
\begin{aligned}
\sigma_{W}^{2} & =\frac{1}{4}\left(\frac{c^{2}}{(1-\rho)}+\frac{c^{2}}{(1+\rho)}\right) \\
& =\frac{c^{2}}{4}\left(\frac{1+\rho+1-\rho}{(1+\rho)(1-\rho)}\right)=\frac{c^{2}}{2\left(1-\rho^{2}\right)}
\end{aligned}
$$

In order for the marginal variances to work out correctly, set equation 12 equal to the marginal variance $\left(\sigma_{1}^{2}=\sigma_{2}^{2} \equiv 1\right)$ and solve for $c$. This yields $c^{2}=2\left(1-\rho^{2}\right)$, so the experimental design will consist of sampling at the major and minor axial points of the ellipse specified by

$$
x^{2}-2 \rho x y+y^{2}=2\left(1-\rho^{2}\right) .
$$

A plot of such ellipses is shown in Figure 2, with correlations ranging from \%ero (a circle) to .9. (For negative correlations, the mirror image of this figure is appropriate.) The standard two-level factorial design (with sampling of both factors at one standard deviation below and one standard deviation above the mean) is the limiting case as $\rho$ approaches zero. However, the design is quite different if correlation is present: the individual factor ranges are larger than

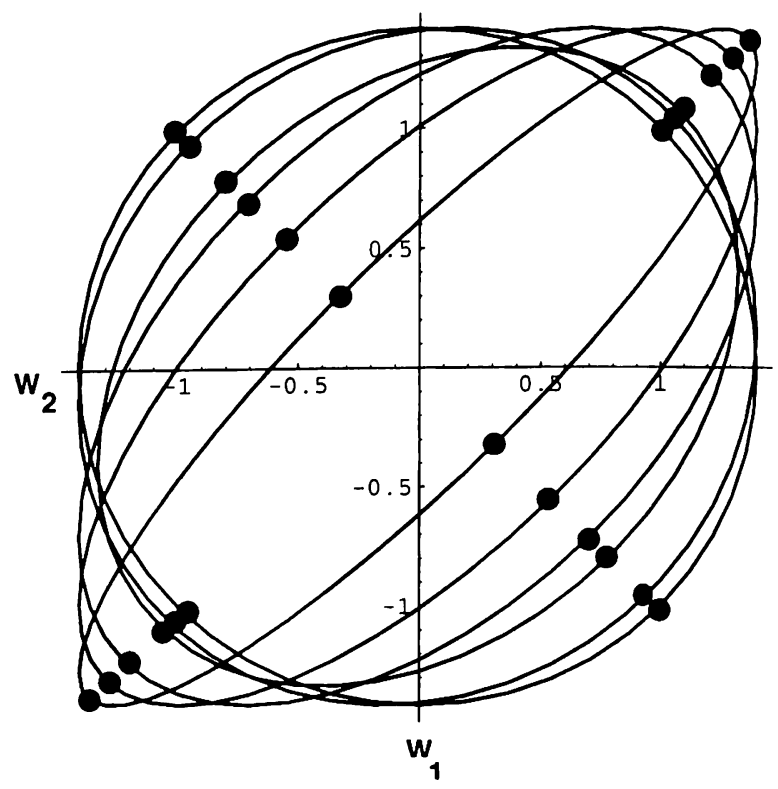

Figure 2: Elliptical Design Points for Two-Level Sanpling Under Correlation, for $\rho=0$ (circle), .1. .3, .5. .7 , and .9

$\mu \pm \sigma$, but the two-dimensional region over which sampling takes place is narrower.

Recall that tolerance design is motivated by a desire to assess system performance across the noise factor space. If sampling at some corners of the range is not practical (or even possible) because of the nature of the response surface, then the methods in Section 2 cannot be used. Reducing the entire range of sampling to allow an orthogonal design puts the design points in a smaller circle within the ellipse, which means more extrapolation is required for predicting system behavior over the relevant noise space. Hence system assessment, even if carried out using equations (3) and (5), could be very inaccurate.

We again turn to the simple circuit example to illustratc the results. When $\rho>0$, we can use a standard factorial design with the convention that for the correlated factors, the coded levels $( \pm 1, \pm 1)$ refer to the design point's quadrant relative to the origin. Actual levels are taken from the ellipse specified in equation (13). Table 4 provides the experimental design, in both coded and natural units.

By applying linear regression to the results in Table 4, we obtain the following metamodel of current:

$$
\begin{gathered}
I \approx \quad 12.393+40.93 L-.9019 R \\
+.0785 V-.0239 f
\end{gathered}
$$

Using equations (3) and (5), we then estimate the response mean and variance. The mean current is approximately 10.133 and the current variance is ap- 
Table 4: Experimental Design for Circuit Example When Correlation $(L, R)=.9$

\begin{tabular}{|c|c|c|c|c|c|}
\hline Coded & \multicolumn{4}{|c|}{ Nat,ural Units } & \multirow[b]{2}{*}{$I$} \\
\hline $\mathrm{L} R \mathrm{R} \mathrm{f}$ & $\mathrm{L}$ & $\mathrm{R}$ & V & $\mathrm{f}$ & \\
\hline---- & .0029 & 8.6216 & 95 & 45 & 10.9693 \\
\hline+--+ & .0043 & 9.6838 & 95 & 55 & 9.6967 \\
\hline-+-+ & .0037 & 10.3162 & 95 & 55 & 9.1389 \\
\hline++-- & .0051 & 11.3784 & 95 & 45 & 9.1548 \\
\hline--++ & .0029 & 8.6216 & 105 & 55 & 12.0973 \\
\hline+-+- & .0043 & 9.6838 & 105 & 45 & 10.7582 \\
\hline-++- & .0037 & 10.3162 & 105 & 45 & 10.1262 \\
\hline++++ & .0051 & 11.3784 & 105 & 55 & 9.1193 \\
\hline
\end{tabular}

proximately .930. Together, the inductor and resistor components account for $81.9 \%$ of the variation in the current, the voltage accounts for $16.6 \%$, and the frequency accounts for $1.5 \%$.

The coefficients of the metamodel terms in in equation (14) differ from those computed using an orthogonal design. The transmitted variances also differ from those computed assuming $\rho=.9$ in in Table 3 . This is to be expected whenever the truc response surface is nonlinear. A linear approximation (and the factors which describe it) will depend a great deal on the area over which the model is fit, which is different for the two experiments. In gencral the difference between using an orthogonal design and an elliptical design to collect data (and considering correlations between variables in the analysis) will be small if the response surface is nearly planar around the region of interest. The circuit current metamodel computed from sampling at elliptical axial points is simpler than that computed from an orthogonal design, but this is not true in general. Also, note that the overall variance estimate obtained from sampling at elliptical axial points is lower than any obtained using the standard factorial design. (This holds even when the full regression metamodel is used.) The estimates in Table 3 are larger by over $18 \%$, even when the correct correlation between $L$ and $R$ of .9 is used for system assessment.

Finally, we remark that it may be possible to simplify the metamodels by removing statistically insignificant terms. For our circuit example, the p-value for $L$ is over .9 for the elliptical experiment, indicating that in practice, the resistance $R$. can be used as a surrogate for the inductance $L$, at least in the context of metamodel building. (Removing insignificant terms from the metamodel means the overall variance estimate drops from .930 to .916.) However, we ad- vise caution in oversimplification when two or more noisc factors are correlated. The sampling schence is not orthogonal, so if $|\rho|$ is near one, then the standard errors of the correlated regression coefficients may be inflated due to multicollinearity. The effect of correlation on the overall variance is computed through the metamodel coefficients, so excluding a miarginally insignificant main effect from the model may have noticeable impacts on system evaluation and noise factor assessment due to the exclusion of interaction terms. We also wish to emphasize that statistical significance in the metamodels does not necessarily imply practical importance. Since the transmitted variances depend on the component variances (and covariances), a term may be statistically significant in the metamodel yet correspond to a factor with transmitted variance near zero.

\section{CONCLUSIONS}

We presented a method for selecting factor settings for two-level experimental designs when pairs of the underlying variables are correlated. We illustrated this using a simple circuit system with four noise fac:tors, including two which were pairwise dependent. Further work is needed to develop designs appropriate for more complicated correlation structures. and to investigate the benefits of incorporating variance reallocation techniques into the designs (Schuben et al., 1992; Donahue et. al. 1992; Tew and Wilson, 1994). Properties such as the bias and mean squared error of the estimators resulting from elliptical axial sampling should also be evaluated more closely. It is possible that other elliptical sampling schemes may be preferred in certain situations.

A final comment is in order regarding the extension of this work to discrete-event simulation. For Monte Carlo experiments, such as the example used in this paper, the transmitted variances for the noise factors sum to the overall system variance. In discreteevent simulation experiments, this is generally not the case: although many noise factors can be explicitly controlled, the system is characterized by some inherent variability which remains unexplained by any noise factors. Sanchez et al. (1994) showed that the tolerance design analysis must be modified to take this into account. In order to reflect the true system variability, they augment the overall system variance estimate of equation (4) by incorporating estimates of the system variability obtained within sinulation runs, and so reflect the true system variability. Such an augmented analysis should also be used when the experimental design includes elliptical axial sampling points. 


\section{ACKNOWLEDGMENTS}

This work was partially supported by a University of Missouri-St. Louis Research Award.

\section{REFERENCES}

Beyer, W. H. 1987. CRC Standard Mathematical Tables, 28th edition. CRC Press, Inc., Boca Raton, Florida.

D'Errico, J. R. and N. A. Zaino. 1988. Statistical Tolerancing Using a Modification of Taguchi's Method, Technometrics, 30(1), 397-405.

Donahuc, J. M., E. R. Houck and R. H. Meyers. 1992. Simulation Designs for Quadratic Response Surface Models in the Presence of Model Misspecification, Management Science, 38(12). 1765-1791.

Dudewicz, E. and S. Mishra. 1988. Modern Mathematical Statistics. John Wiley and Sons, Inc., New York.

Myers, R. H., A. I. Khuri and G. Vining. 1992. Response Surface Alternatives to the Taguchi Robust Parameter Design Approach, The American Statistician, 46(2), 131-139.

Ramberg, J. S., S. M. Sanchez, P. J. Sanchez and L. W. Hollick. 1991. Designing Simulation Experiments: Taguchi Methods and Response Surface Metamodels, Proceedings of the 1994 Winter Simulation Conference, eds. B. L. Nelson, W. D. Kelton and G. M. Clark, 167-176. Institute of Electrical and Electronic Engineers, Phoenix, Arizona,.

Sanchez, S. M., J. S. Ramberg, J. Fiero and J. J. Pignatiello, Jr. 1993. Quality by Design. Chapter 10 in Concurrent Engineering: Automation, Tools, and Techniques, A. Kusiak (Ed.). John Wiley and Sons, NY, 235-286.

Sanchez, S. M. 1994. A Robust Design Tutorial, Proceedings of the 1994 Winter Simulation Conference, eds. J. D. Tew, M. S. Manivannan, D. A. Sadowski and A. F. Seila, Institute of Electrical and Electronic Engineers, Orlando, Florida.

Sanche\%, S. M., P. J. Sanchez and J. S. Ramberg. 1994. A Robust Design Framework for Discrete Event Simulation, Working paper 94-06-08, School of Business Administration, University of Missouri-St. Louis.

Schruben, L. W., S. M. Sanchez, P. J. Sanchez and V. A. Critrom. 1992. Variance Reallocation in Taguchi's Robust Design Framework, Proceedings of the 1992 Winter Simulation Conference, eds. J. J. Swain. D. Goldsman, R. C. Crain and J. R. Wilson, 548-556, Washington, D. C.

Smith, L. D., S. M. Sanchez and E. C. Lawrence. 1994. A Comprehensive Model for Managing
Credit Risk and Forecasting Losses on Home Mortgage Portfolios. Working Paper 94-06-04, Center for Business and Industrial Studies, School of Business Administration, University of MissouriSt. Louis.

Taguchi, G. 1986. Introduction to Quality Engineering, UNIPUB/Krauss International: White Plains, New York.

Taguchi, G. 1987. System of Experimental Design, Vols. 1 and 2. UNIPUB/Krauss International: White Plains, New York.

Tew, J. D. and Wilson, J. R. 1994. Estimating Simulation Metamodels Using Combined CorrelationBased Reduction Techniques. IIE Transactions. 26(3), 2-16.

\section{AUTHOR BIOGRAPHY}

SUSAN M. SANCHEZ is an associate professor in Management Science and Information Systems at the University of Missouri-St. Louis. Her research interests include the design and analysis of simulation experiments, selection, and data-intensive statistical methods in business and manufacturing. She is a member of INFORMS (formally ORSA and TIMS). ASA, and ASQC. 